\title{
Estimation and prediction for the Kumaraswamy-inverse Rayleigh distribution based on records
}

\author{
Mohamed A. Hussian ${ }^{1}$, Essam A. Amin ${ }^{2}$ \\ ${ }^{I}$ Department of statistics and operations research, faculty of science, King Saud University. \\ ${ }^{2}$ Mathematics and Statistics Department, College of Science, Al-Imam Mohammad Ibn Saud Islamic University. \\ *Corresponding author E-mail: msabry@ksu.edu.sa
}

Copyright $\odot 2014$ Mohamed A. Hussian and Essam A. Amin. This is an open access article distributed under the Creative Commons Attribution License, which permits unrestricted use, distribution, and reproduction in any medium, provided the original work is properly cited.

\begin{abstract}
In this paper, estimators for the parameters of the Kumaraswamy-inverse Rayleigh distribution based on record values are obtained. These estimators are derived using the maximum likelihood and Bayesian methods. The Bayesian estimators are derived under the well-known squared error (SE) loss function. Prediction of the future sth record value is derived using the maximum likelihood and Bayesian methods. Simulation study is conduct to illustrate the findings.

Keywords: Kumaraswamy, Inverse Rayleigh, record values, Bayes estimator, squared error loss function prediction of future record values, Bayes estimation; maximum likelihood.
\end{abstract}

\section{Introduction}

Record values and associated statistics are of great importance to scientists and engineers in several real life problems involving weather, economic, and support data. For example, predicting the flood level of a river that is greater than the previous ones is of importance to climatologists and hydrologists. Predicting the magnitude of an earthquake which has a greater magnitude than the previous ones, in a given region, is of importance to seismologists as well. Moreover, record values are also important to ordinary people regarding all kinds of strange and extreme phenomenon and talents. While a lot of work has been done on characterizations, asymptotic theory and generalizations, not much has been done on statistical inference based on record values. Chandler [1] introduced the theory of record values for the first time, and since then, many authors have studied record values and the associated statistics. Interested readers may refer to [2], [7]. Balakrishnan et al., [8], [10] have established some recurrence relations for the moments of record values from Gumbel, generalized Pareto, and exponential distributions respectively and Balakrishnan et al., [11] discussed some inferential methods based on record values from exponential, Gumbel, Weibull and logistic distributions respectively. Furthermore, several authors have studied distribution characteristics based on record value. For example, Selim, [12] studied Bayesian estimation of two parameter of bathtub-shape lifetime distribution based on record values and Nader et. al., [13] inferentially studied record values from the Kumaraswamy distribution. Moreover, Amini and Balakrishnan, [14] derived exact distribution-free confidence intervals for quantiles of the population of ordered records and exact prediction intervals for future record values. In 2014, Juhas and Skrivankova [15] made several characterizations of general classes of distributions using the independence of suitable transformations of records in a sequence of independent, identically distributed random variables with examples of Gumbel, Frechet, Weibull, exponential and lognormal distributions. For complete review see [2], [3], [10], and [16].

Let $X_{1}, X_{2}, \ldots$ be a sequence of independent and identically distributed (i.i.d.) random variables with the common cumulative distribution function $(c d f) F(x ; \theta)$ and probability density function $(p d f) f(x ; \theta)$ where $\theta$ is the parameter vector. An observation $X_{j}$ is called an upper record value if it exceeds all previous observations. Thus $X_{j}$ is an upper record value if $X_{j}>X_{i}$ for all $i<j$. 
In this paper, we use Bayesian and non-Bayesian methods for the estimation of the unknown parameters and prediction of the $s$ th future record values, when $m$ records have been observed from the Kumaraswamy-inverse Rayleigh $(K w-I R)$ distribution [17]. The $K w-I R$ has the following $c d f$ and $p d f$ for $X>0$ :

$F(x)=1-\left[1-\exp \left(-\lambda x^{-2}\right)\right]^{\alpha}$,

and

$f(x)=2 \alpha \lambda x^{-3} \exp \left(-\lambda x^{-2}\right)\left[1-\exp \left(-\lambda x^{-2}\right)\right]^{\alpha-1}$,

respectively, where $\lambda>0$ and $\alpha>0$ are scale and shape parameters respectively.

The rest of the paper is organized as follows: In section 2, Maximum likelihood (ML) estimators of the parameters of the $K w$-IR distribution based on record values and prediction of the sth future record value are derived. Section 3 is devoted to Bayesian methods. Results of a Monte Carlo simulation study conducted to evaluate the performance of these estimators compared to the ML estimators and the Bayesian estimators as well as prediction of the sth future value are provided in Section 4.

\section{Likelihood methods}

\subsection{Maximum likelihood estimation method}

Consider the vector of first observed $m$ record values $\boldsymbol{r}=\left(r_{1}, r_{2}, \ldots, r_{m}\right)$ drawn from a population with pdf. The joint pdf of the first $m$ upper record values [7] is given by

$f(r ; \theta)=\prod_{i=1}^{m-1} h\left(r_{i} ; \theta\right) f\left(r_{m} ; \theta\right)$

where

$h\left(r_{i} ; \theta\right)=\frac{f\left(r_{i} ; \theta\right)}{1-F\left(r_{i} ; \theta\right)}$,

and $-\infty<r_{1}<r_{2}<\ldots<r_{m}<\infty$, and $\theta$ is the parameter vector.

Suppose we observed the first $m$ upper record values $R_{1}=r_{1}, R_{2}=r_{2}, \ldots, R_{m}=r_{m}$ from the $K w-I R$ distribution with $c d f$ and $p d f$ given by Eqs. (1) and (2) respectively. Then the likelihood function is given by

$$
\begin{aligned}
L(\boldsymbol{r} ; \alpha, \lambda) & =\prod_{i=1}^{m-1} \frac{f\left(r_{i} ; \alpha, \lambda\right)}{1-F\left(r_{i} ; \alpha, \lambda\right)} f\left(r_{m} ; \alpha, \lambda\right) \\
& =2^{m} \alpha^{m} \lambda^{m} \exp \left(-\sum_{i=1}^{m} \lambda r_{i}^{-3}\right)\left[A_{m}\right]^{\alpha} \prod_{i=1}^{m} \frac{r_{i}^{-3}}{A_{i}},
\end{aligned}
$$

where $A_{i}(\lambda)=1-\exp \left(-\lambda r_{i}^{-2}\right), i=1,2,3, \ldots$, and the log-likelihood function will be

$\ln L(\boldsymbol{r} ; \alpha, \lambda) \propto m \ln \alpha+m \ln \lambda-\lambda \sum_{i=1}^{m} r_{i}^{-3}+\alpha \ln A_{m}(\lambda)-\sum_{i=1}^{m} \ln A_{i}(\lambda)$.

The estimators $\hat{\alpha}$ and $\hat{\lambda}$ of the parameters $\alpha$ and $\lambda$ respectively can be then obtained as the solution of the likelihood equations

$\frac{m}{\hat{\alpha}}+\ln A_{m}(\hat{\lambda})=0$

$\frac{m}{\hat{\lambda}}-\sum_{i=1}^{m} r_{i}^{-3}+\frac{\hat{\alpha} r_{m}^{-2}\left(1-A_{m}(\lambda)\right)}{A_{m}(\hat{\lambda})}+\sum_{i=1}^{m} \frac{r_{i}^{-2}\left(1-A_{i}(\hat{\lambda})\right)}{A_{i}(\hat{\lambda})}=0$.

From Eq. (7), we have

$$
\hat{\alpha}=-\frac{m}{\ln A_{m}(\hat{\lambda})} \text {. }
$$

where $\hat{\lambda}$ is the solution of the nonlinear equation

$$
\frac{m}{\hat{\lambda}}-\sum_{i=1}^{m} r_{i}^{-3}-\frac{m r_{m}{ }^{-2}\left(1-A_{m}(\hat{\lambda})\right)}{A_{m}(\hat{\lambda}) \ln A_{m}(\hat{\lambda})}+\sum_{i=1}^{m} \frac{r_{i}^{-2}\left(1-A_{i}(\hat{\lambda})\right)}{A_{i}(\hat{\lambda})}=0 .
$$

Several numerical techniques can be used to solve this system of nonlinear equations.

\subsection{Maximum likelihood prediction method}

Consider that, the first $m$ upper records, have been observed from $K w$ - $I R$ distribution with parameters $\alpha$ and $\lambda$ and let $R_{s}$, where $s>m$ be the $s$ th record value. To find a prediction value for $R_{s}$ say $r_{s}$, Basak and Balakrishinan [18], proposed a joint predictive function based on the likelihood function of the form 
$L\left(r_{s} ; \alpha, \lambda, \boldsymbol{r}\right)=\frac{1}{\Gamma(s-m)}\left[\ln \frac{1-F\left(r_{m} ; \alpha, \lambda\right)}{1-F\left(r_{s} ; \alpha, \lambda\right)}\right]^{s-m-1} \prod_{i=1}^{m} \frac{f\left(r_{i} ; \alpha, \lambda\right)}{1-F\left(r_{i} ; \alpha, \lambda\right)} f\left(r_{s} ; \alpha, \lambda\right)$.

In the case of the $K w$-IR distribution, Eq. (11) will lead to

$L\left(r_{s} ; \alpha, \lambda, \boldsymbol{r}\right) \propto \lambda^{m+1} \alpha^{s}\left(1-A_{s}(\lambda)\right)\left[A_{m}(\lambda)\right]^{\alpha-1} \prod_{i=1}^{m} \frac{r_{i}^{-3}\left(1-A_{i}(\lambda)\right)}{A_{i}(\lambda)} \times\left[\ln \frac{A_{m}(\lambda)}{A_{s}(\lambda)}\right]^{s-m-1}$

The associated log-likelihood will be given by

$$
\begin{aligned}
\ln L\left(r_{s} ; \alpha, \lambda, \boldsymbol{r}\right) \propto & (m+1) \ln \lambda+s \ln \alpha_{s}-\lambda r_{s}^{-2}+(\alpha-1) \ln \left[A_{s}(\lambda)\right]-\lambda \sum_{i=1}^{m} r_{i}^{-2} \\
& -\sum_{i=1}^{m} \ln A_{i}(\lambda)+(s-m-1) \ln \left(\ln A_{m}(\lambda)-\ln A_{s}(\lambda)\right)
\end{aligned}
$$

where $0<r_{1}<r_{2}<\ldots<r_{m}<r_{s}$. In order to find the estimators of $\alpha, \lambda$ and, we need to solve the log-likelihood equations with respect to $\alpha, \lambda$ and. This will lead to the following system of nonlinear equations

$$
\begin{aligned}
& \frac{s}{\alpha}+\ln A_{s}(\lambda)=0 \\
& \frac{m+1}{\lambda}-r_{s}^{-2}+\sum_{i=1}^{m} r_{i}^{-2}+\frac{(\alpha-1) r_{s}^{-2}\left(1-A_{s}(\lambda)\right)}{A_{s}(\lambda)}+\sum_{i=1}^{m} \frac{r_{i}^{-2}\left(1-A_{i}(\lambda)\right)}{\ln A_{i}(\lambda)} \\
& \quad+\frac{(s-m-1)}{\left(\ln A_{m}(\lambda)-\ln A_{s}(\lambda)\right)}\left(\frac{r_{m}^{-2}\left(1-A_{m}(\lambda)\right)}{A_{m}(\lambda)}-\frac{r_{s}^{-2}\left(1-A_{s}(\lambda)\right)}{A_{s}(\lambda)}\right)=0, \\
& \frac{-3}{r_{s}}+2 \lambda r_{s}^{-3}-\frac{2 \lambda(\alpha-1) r_{s}^{-3}\left(1-A_{s}(\lambda)\right)}{A_{s}(\lambda)}+\frac{2 \lambda(s-m-1)}{\left(\ln A_{m}(\lambda)-\ln A_{s}(\lambda)\right)}\left(\frac{r_{s}^{-3}\left(1-A_{s}(\lambda)\right)}{A_{s}(\lambda)}\right)=0 .
\end{aligned}
$$

From Eq. (14), we have

$$
\hat{\alpha}=-\frac{m}{\ln A_{s}(\tilde{\lambda})}
$$

Substituting Eq. (17) into Eqs. (15) and (16), we have

$$
\begin{gathered}
\frac{m+1}{\hat{\lambda}}-r_{s}^{-2}+\sum_{i=1}^{m} r_{i}^{-2}-\left(\frac{m}{\ln A_{s}(\hat{\lambda})}+1\right) r_{s}^{-2} \frac{\left(1-A_{s}(\hat{\lambda})\right)}{A_{s}(\hat{\lambda})}+\sum_{i=1}^{m} \frac{r_{i}^{-2}\left(1-A_{i}(\hat{\lambda})\right)}{\ln A_{i}(\hat{\lambda})} \\
+\frac{(s-m-1)\left(\frac{r_{m}^{-2}\left(1-A_{m}(\tilde{\lambda})\right)}{A_{m}(\tilde{\lambda})}-\frac{r_{s}^{-2}\left(1-A_{s}(\tilde{\lambda})\right)}{A_{s}(\tilde{\lambda})}\right)}{\ln \left[1-\exp \left(-\hat{\lambda} r_{m}^{-2}\right)\right]-\ln \left[1-\exp \left(-\hat{\lambda} r_{s}^{-2}\right)\right]}=0, \\
\frac{-3}{r_{s}}+\frac{2 \tilde{\lambda}}{r_{s}^{3}}+2 \tilde{\lambda}\left(\frac{m}{\ln A_{s}(\tilde{\lambda})}+1\right) \frac{\left(1-A_{s}(\tilde{\lambda})\right)}{r_{s}^{3} A_{s}(\tilde{\lambda})}+\frac{2 \tilde{\lambda}(s-m-1)}{\ln A_{m}(\tilde{\lambda})-\ln A_{s}(\tilde{\lambda})}\left(\frac{r_{s}^{-3}\left(1-A_{s}(\tilde{\lambda})\right)}{A_{s}(\tilde{\lambda})}\right)=0
\end{gathered}
$$

Solving the nonlinear Eqs. (18) and (19) numerically, we will be able to estimate the predictive value of the $s$ th record value based on $K w-I R$ distribution.

\section{Bayesian methods}

\subsection{Bayesian estimation method}

Assume that the parameters $\alpha$ and $\lambda$ are random variables with a joint bivariate prior density function [18] of the form $\pi(\alpha, \lambda)=\pi_{1}(\alpha \mid \lambda) \pi_{2}(\lambda)$

where

$$
\pi_{1}(\alpha \mid \lambda)=\frac{\lambda^{a_{1}+1}}{\Gamma\left(a_{1}+1\right) b_{1}^{a_{1}+1}} \alpha^{a_{1}} \exp \left(-\frac{\alpha \lambda}{b_{1}}\right),
$$

and

$$
\pi_{2}(\lambda)=\frac{1}{\Gamma\left(a_{2}\right) b_{2}^{a_{2}}} \lambda^{a_{2}-1} \exp \left(-\frac{\lambda}{b_{2}}\right),
$$

where the hyper-parameters $a_{1}>-1, a_{2}>0, b_{1}>0$ and $b_{2}>0$. Thus, the bivariate prior density of $\alpha$ and $\lambda$ is given by 
$\pi(\alpha, \lambda)=\frac{\lambda^{a_{1}+a_{2}} \alpha^{a_{1}}}{\Gamma\left(a_{1}+1\right) \Gamma\left(a_{2}\right) b_{1}^{a_{1}+1} b_{2}^{a_{2}}} \exp \left(-\lambda\left[\frac{1}{b_{2}}+\frac{\alpha}{b_{1}}\right]\right)$.

From Eqs. (5) And (23), the joint posterior distribution function of both $\alpha$ and $\lambda$ is given by

$$
\begin{aligned}
\pi(\alpha, \lambda \mid \boldsymbol{r}) & =\frac{L(\alpha, \lambda ; \boldsymbol{r}) \pi(\alpha, \lambda)}{\int_{0}^{\infty} \int_{0}^{\infty} L(\alpha, \lambda ; \boldsymbol{r}) \pi(\alpha, \lambda) d \alpha d \lambda} \\
& =\frac{\lambda^{m+a_{1}+a_{2}} \alpha^{m+a_{1}}}{K \Gamma\left(m+a_{1}+1\right)} \exp \left(-\lambda\left\{\frac{1}{b_{2}}+\sum_{i=1}^{m} r_{i}^{-2}\right\}\right) \exp \left(-\alpha\left\{\frac{1}{b_{1}}-\ln A_{m}(\lambda)\right\}\right) \times \exp \left(-\left[\sum_{i=1}^{m} \ln A_{i}(\lambda)-\sum_{i=1}^{m} \ln r_{i}^{-3}\right]\right)
\end{aligned}
$$

where

$$
K=\int_{0}^{\infty} \frac{\lambda^{m+a_{1}+a_{2}}}{\left(\frac{1}{b_{1}}-\ln A_{m}(\lambda)\right)^{m+a_{1}+1}} \exp \left(-\lambda\left\{\frac{1}{b_{2}}+\sum_{i=1}^{m} r_{i}^{-2}\right\}-\left[\sum_{i=1}^{m} \ln A_{i}(\lambda)-\sum_{i=1}^{m} \ln r_{i}^{-3}\right]\right) d \lambda .
$$

Under the well-known squared error (SE) loss function, the Bayes estimators of $\alpha$ and $\lambda$ are the expected value based on their marginal posterior distributions. This will lead to

$$
\hat{\hat{\alpha}}=E(\alpha \mid \boldsymbol{r})=\left(m+a_{1}+1\right) \frac{K_{1}}{K},
$$

and

$$
\hat{\hat{\lambda}}=E(\lambda \mid \boldsymbol{r})=\frac{K_{2}}{K \Gamma\left(m+a_{1}+1\right)},
$$

where

$$
K_{1}=\int_{0}^{\infty} \frac{\lambda^{m+a_{1}+a_{2}}}{\left(\frac{1}{b_{1}}-\ln A_{m}(\lambda)\right)^{m+a_{1}+2}} \exp \left(-\lambda\left\{\frac{1}{b_{2}}+\sum_{i=1}^{m} r_{i}^{-2}\right\}-\left[\sum_{i=1}^{m} \ln A_{i}(\lambda)-\sum_{i=1}^{m} \ln r_{i}^{-3}\right]\right) d \alpha,
$$

and

$$
K_{2}=\int_{0}^{\infty} \frac{\lambda^{m+a_{1}+a_{2}+1}}{\left(\frac{1}{b_{1}}-\ln A_{m}(\lambda)\right)^{m+a_{1}+1}} \exp \left(-\lambda\left\{\frac{1}{b_{2}}+\sum_{i=1}^{m} r_{i}^{-2}\right\}-\left[\sum_{i=1}^{m} \ln A_{i}(\lambda)-\sum_{i=1}^{m} \ln r_{i}^{-3}\right]\right) d \lambda
$$

\subsection{Bayesian prediction method}

Consider that the first $m$ upper records have been observed from the $K w$ - $I R$ distribution. Let, where $s>m$ the sth upper record value be. The aim is to predict $R_{s}$ given $\boldsymbol{r}$. This is done using the conditional density function of $R_{s}$ given $\boldsymbol{r}$ [5, 19] which is given by

$f\left(r_{s} \mid \boldsymbol{r}, \theta\right)=\frac{1}{\Gamma(s-m)}\left[\ln \frac{1-F\left(r_{m} ; \alpha, \lambda\right)}{1-F\left(r_{s} ; \alpha, \lambda\right)}\right]^{s-m-1} \frac{f\left(r_{s} ; \theta\right)}{1-F\left(r_{m} ; \theta\right)}$,

and the Bayes predictive density function

$h\left(r_{s} \mid \boldsymbol{r}, \theta\right)=\int_{\theta} f\left(r_{s} ; \underline{\boldsymbol{r}}, \theta\right) \pi(\theta \mid \boldsymbol{r}) d \theta$.

In the case of the $K w$-IR distribution, the Bayes predictive density of $R_{s}$ given $r$ is

$$
\begin{aligned}
h\left(r_{s} \mid \boldsymbol{r}, \alpha, \lambda\right)=\int_{\alpha} \int_{\lambda} & \frac{\left.2^{m} \lambda^{m+a_{1}+a_{2}+1} \alpha^{m+a_{1}+1}\left(1-A_{s}(\lambda)\right)\left[A_{s}(\lambda)\right)\right]^{\alpha-1}}{r_{s}^{3} \Gamma(s-m)\left[A_{m}(\lambda)\right]^{\alpha} \Gamma\left(a_{1}+1\right) \Gamma\left(a_{2}\right) b_{1}^{a_{1}+1} b_{2}{ }^{a_{2}} \Gamma\left(m+a_{1}+1\right)} \\
& \times \exp \left(-\lambda\left\{\frac{1}{b_{2}}+\sum_{i=1}^{m} r_{i}^{-2}\right\}\right) \exp \left(-\alpha\left\{\frac{1}{b_{1}}-\ln A_{m}(\lambda)\right\}\right)\left(\alpha \ln \frac{\left.A_{m}(\lambda)\right)}{A_{s}(\lambda)}\right)^{s-m-1} \\
& \times \exp \left(\sum_{i=1}^{m} \ln \frac{r_{i}^{-2}}{A_{i}(\lambda)}\right)\left(\frac{1}{K}\right) d \lambda d \alpha
\end{aligned}
$$

that can be written as,

$h\left(r_{s} \mid \boldsymbol{r}, \theta\right)=\frac{1}{r_{s}{ }^{2} b_{1}^{a_{1}+1} b_{2}^{a_{2}} \Gamma\left(a_{1}+1\right) \Gamma\left(a_{2}\right) \mathrm{B}\left(s-m, m+a_{1}+1\right)}\left(\frac{1}{K}\right) \Psi\left(\boldsymbol{r}, r_{s}\right)$,

where 
$\Psi\left(\boldsymbol{r}, r_{s}\right)=2^{m} \int_{0}^{\infty} \frac{\lambda^{m+a_{1}+a_{2}+1} \exp \left(-\lambda\left\{\frac{1}{b_{2}}-r_{s}^{-2}+\sum_{i=1}^{m} r_{i}^{-2}\right\}\right)\left[\ln \frac{A_{m}(\lambda)}{A_{s}(\lambda)}\right]^{s-m-1}}{\left\{\frac{\lambda}{b_{1}}-\ln A_{m}(\lambda)\right\}^{s+a_{1}+1} \exp \left[\sum_{i=1}^{m} \ln \frac{r_{i}^{-2}}{A_{i}(\lambda)}+\ln A_{s}(\lambda)\right]} d \lambda$.

and $B\left(s-m, m+a_{1}+1\right)$ is the beta function. Based on the squared error (SE) loss function the Bayesian prediction of the sth upper record value is given by the expected value of the Bayes predictive density function. This will lead to the estimator $r_{s(B S)}$ where

$$
r_{s(B S)}=E\left(r_{s} \mid \mathrm{r}\right)=\frac{1}{b_{1}^{a_{1}+1} b_{2}^{a_{2}} \Gamma\left(a_{1}+1\right) \Gamma\left(a_{2}\right) \mathrm{B}\left(s-m, m+a_{1}+1\right)} \int_{r_{m}}^{\infty} \frac{\Psi\left(\mathrm{r}, r_{s}\right)}{K r_{s}} d r_{s}
$$

\section{Numerical example}

In this section, numerical illustrations are made to assess the statistical performances of the ML and Bayes estimators of both shape and scale parameters and the prediction of the sth record value. Bayes estimators for the parameters $\alpha$ and $\lambda$ and the predicted $s$ th record value are obtained under the SE loss function using informative $\left(a_{1}, a_{2}, b_{1}, b_{2}>0\right.$ for example, $\left.a_{1}=a_{2}=3 \quad b_{1}=b_{2}=2\right)$, and non-informative priors $\left(a_{1}=a_{2}=b_{1}=b_{2}=0.0001\right)$, see [20]. The performance assessment is made by comparing the biases and the mean squared errors (MSE) of the estimators of $\alpha$ and $\lambda$ and the future $s$ th record value $r_{s}$. The simulations are made using MATHEMATICA v.8 for several combinations of the parameters $n, m, s, \alpha$ and $\lambda$. The random samples of $K w$-IR are generated using the form

$x=\left(-\frac{\lambda}{\log \left[1-u^{1 / \alpha}\right]}\right)^{0.5}, \quad x \geq 0, \quad \lambda, \alpha>0$

Where $0<u<1$ is a uniform random variable. After that, the first $12^{\text {th }}$ upper record values are observed as in Table 1 . Different simulations are based on 1000 replications. The results are shown in Tables 2-5. Tables 6 and 7 represents the efficiency of Bayesian estimators with respects to the ML estimators of the shape and scale parameters, where the efficiency of a parameter $\theta_{2}$ with respect to a parameter $\theta_{1}$ is given by

eff $\left(\hat{\theta}_{1}, \hat{\theta}_{2}\right)=\frac{\operatorname{MSE}\left(\hat{\theta}_{1}\right)}{\operatorname{MSE}\left(\hat{\theta}_{2}\right)}$

Table 1: Samples of upper records for different parameter values

\begin{tabular}{ccccccccccccc}
\hline$(\alpha, \lambda)$ & 1 & 2 & 3 & 4 & 5 & 6 & 7 & 8 & 9 & 10 & 11 & 12 \\
\hline$(0.5,0.5)$ & 0.4214 & 0.4785 & 0.4805 & 0.4918 & 0.4957 & 0.5051 & 0.5162 & 0.5177 & 0.5219 & 0.5295 & 0.5302 & 0.5334 \\
$(1,0.5)$ & 0.4198 & 0.5560 & 0.5653 & 0.6254 & 0.6482 & 0.6699 & 0.6701 & 0.6804 & 0.6848 & 0.6878 & 0.7104 & 0.7331 \\
$(2,0.5)$ & 0.3825 & 0.4238 & 0.4252 & 0.4329 & 0.4355 & 0.4419 & 0.4493 & 0.4503 & 0.4531 & 0.4581 & 0.4585 & 0.4607 \\
$(0.5,1)$ & 0.5960 & 0.6768 & 0.6796 & 0.6956 & 0.7009 & 0.7143 & 0.7300 & 0.7321 & 0.7382 & 0.7489 & 0.7497 & 0.7544 \\
$(1,1)$ & 0.5665 & 0.6346 & 0.6369 & 0.6500 & 0.6544 & 0.6652 & 0.6779 & 0.6796 & 0.6844 & 0.6930 & 0.6937 & 0.6974 \\
$(2,1)$ & 0.5409 & 0.5993 & 0.6012 & 0.6123 & 0.6160 & 0.6250 & 0.6355 & 0.6369 & 0.6409 & 0.6479 & 0.6485 & 0.6515 \\
\hline
\end{tabular}

Table 2: Biases of Maximum Likelihood and Bayes estimates for $\alpha$ and $\lambda$ and the future $s$ th record value when $\lambda=0.5$

\begin{tabular}{clccccccccc}
\hline$\alpha$ & $(m, s)$ & \multicolumn{3}{c}{ Maximum Likelihood } & \multicolumn{3}{c}{ Non-informative Bayes } & \multicolumn{2}{c}{ Informative Bayes } \\
\hline & & $\tilde{\alpha}$ & $\tilde{\lambda}$ & $r_{s}$ & $\hat{\hat{\alpha}}$ & $\hat{\hat{\lambda}}$ & $r_{s(B S)}$ & $\hat{\hat{\alpha}}$ & $\hat{\hat{\lambda}}$ & $r_{s(B S)}$ \\
0.5 & $(5,7)$ & 0.4848 & 0.2548 & 0.2880 & 0.2676 & 0.1757 & 0.2327 & 0.1336 & 0.1320 & 0.1420 \\
& $(7,9)$ & 0.3336 & 0.1838 & 0.2143 & 0.1279 & 0.1462 & 0.1518 & 0.1198 & 0.1006 & 0.1101 \\
& $(10,12)$ & 0.1108 & 0.1229 & 0.1774 & 0.1005 & 0.1138 & 0.1096 & 0.0938 & 0.0787 & 0.0688 \\
1 & $(5,7)$ & 0.5097 & 0.2679 & 0.3028 & 0.2813 & 0.1847 & 0.2446 & 0.1584 & 0.1388 & 0.1493 \\
& $(7,9)$ & 0.3507 & 0.1932 & 0.2253 & 0.1345 & 0.1537 & 0.1596 & 0.1254 & 0.1058 & 0.1157 \\
& $(10,12)$ & 0.1165 & 0.1292 & 0.1865 & 0.1057 & 0.1196 & 0.1152 & 0.1186 & 0.0827 & 0.0723 \\
2 & $(5,7)$ & 0.5922 & 0.3113 & 0.3518 & 0.3268 & 0.2146 & 0.2842 & 0.1743 & 0.1613 & 0.1735 \\
& $(7,9)$ & 0.4075 & 0.2245 & 0.2618 & 0.1563 & 0.1786 & 0.1854 & 0.1446 & 0.1229 & 0.1344 \\
& $(10,12)$ & 0.1354 & 0.1501 & 0.2167 & 0.1228 & 0.1390 & 0.1338 & 0.1295 & 0.0961 & 0.0840 \\
\hline
\end{tabular}

\section{Results and discussion}

From tables 2-5, one can see that the biases and MSEs of both Bayes estimators of the shape and scale parameters and the predicted values of the future sth record value are smaller than the corresponding ML estimators and predicted values of the future sth record value. Moreover, for fixed shape and scale parameter values, the biases and MSEs of the 
estimators and the predicted values of the future sth record value based on all methods decreases as the size of the record samples increases. On the other hand, for fixed value of the scale parameter, the biases and MSEs of the estimators of both the shape and scale parameters and the future $s$ th record value based on all methods increases as the value of the shape parameter $\alpha$ increases. In addition, when $\lambda$ increases, improved estimates and predicted sth value are obtained for all methods. Altogether, Tables 6 and 7 emphasize that results based on informative and noninformative Bayesian estimation methods are superior to that of the ML estimation method through the efficiency of Bayes estimators with respect to ML estimators and by looking deeply, Bayes estimators and future sth prediction value based on informative priors are more efficient than the non-informative ones. In conclusion, Bayesian estimation method based on informative priors are recommended for estimation and prediction of future record values for the Kumaraswamy inverse Rayleigh distribution

Table 3: Biases of Maximum Likelihood and Bayes estimates for $\alpha$ and $\lambda$ and the future sth record value when $\lambda=1$

\begin{tabular}{lllllcccccc}
\hline$\alpha$ & $(m, s)$ & \multicolumn{3}{c}{ Maximum Likelihood } & \multicolumn{3}{c}{ Non-informative Bayes } & \multicolumn{2}{c}{ Informative Bayes } \\
\hline \multirow{4}{*}{0.5} & & $\tilde{\alpha}$ & $\tilde{\lambda}$ & $r_{s}$ & $\hat{\alpha}$ & $\hat{\hat{\lambda}}$ & $r_{s(B S)}$ & $\hat{\alpha}$ & $\hat{\lambda}$ & $r_{s(B S)}$ \\
& $(5,7)$ & 0.4173 & 0.2193 & 0.2479 & 0.2191 & 0.1439 & 0.1905 & 0.1290 & 0.1275 & 0.1371 \\
& $(7,9)$ & 0.2871 & 0.1582 & 0.1844 & 0.1047 & 0.1197 & 0.1243 & 0.1157 & 0.0971 & 0.1063 \\
1 & $(10,12)$ & 0.0954 & 0.1058 & 0.1527 & 0.0823 & 0.0932 & 0.0897 & 0.0906 & 0.0760 & 0.0664 \\
& $(5,7)$ & 0.4387 & 0.2306 & 0.2606 & 0.2303 & 0.1512 & 0.2003 & 0.1530 & 0.1340 & 0.1442 \\
& $(7,9)$ & 0.3019 & 0.1663 & 0.1939 & 0.1101 & 0.1258 & 0.1307 & 0.1211 & 0.1022 & 0.1117 \\
& $(10,12)$ & 0.1003 & 0.1112 & 0.1605 & 0.0865 & 0.0979 & 0.0943 & 0.1145 & 0.0799 & 0.0698 \\
2 & $(5,7)$ & 0.5097 & 0.2679 & 0.3028 & 0.2676 & 0.1757 & 0.2327 & 0.1683 & 0.1558 & 0.1675 \\
& $(7,9)$ & 0.3507 & 0.1932 & 0.2253 & 0.1280 & 0.1462 & 0.1518 & 0.1396 & 0.1187 & 0.1298 \\
& $(10,12)$ & 0.1165 & 0.1292 & 0.1865 & 0.1005 & 0.1138 & 0.1095 & 0.1250 & 0.0928 & 0.0811 \\
\hline
\end{tabular}

Table 4: MSEs of Maximum Likelihood and Bayes estimates for $\alpha$ and $\lambda$ and the future $s$ th record value when $\lambda=0.5$

\begin{tabular}{|c|c|c|c|c|c|c|c|c|c|c|}
\hline \multirow[t]{2}{*}{$\alpha$} & \multirow[t]{2}{*}{$(m, s)$} & \multicolumn{3}{|c|}{ Maximum Likelihood } & \multicolumn{3}{|c|}{ Non-informative Bayes } & \multicolumn{3}{|c|}{ Informative Bayes } \\
\hline & & $\tilde{\alpha}$ & $\tilde{\lambda}$ & $r_{s}$ & $\hat{\hat{\alpha}}$ & $\hat{\hat{\lambda}}$ & $r_{s(B S)}$ & $\hat{\hat{\alpha}}$ & $\hat{\hat{\lambda}}$ & $r_{s(B S)}$ \\
\hline \multirow[t]{3}{*}{0.5} & $(5,7)$ & 0.3829 & 0.3259 & 0.3003 & 0.3053 & 0.2668 & 0.2491 & 0.25 & 0.2185 & 0.2039 \\
\hline & $(7,9)$ & 0.3255 & 0.2905 & 0.2658 & 0.2542 & 0.2297 & 0.2144 & 0.2081 & 0.188 & 0.1755 \\
\hline & $(10,12)$ & 0.2796 & 0.2652 & 0.2364 & 0.2035 & 0.1977 & 0.1845 & 0.1666 & 0.1618 & 0.1511 \\
\hline \multirow[t]{3}{*}{1} & $(5,7)$ & 0.4155 & 0.3721 & 0.3295 & 0.3374 & 0.31 & 0.2753 & 0.2763 & 0.2538 & 0.2254 \\
\hline & $(7,9)$ & 0.3532 & 0.3298 & 0.2894 & 0.281 & 0.2668 & 0.2369 & 0.23 & 0.2185 & 0.194 \\
\hline & $(10,12)$ & 0.2985 & 0.3052 & 0.2589 & 0.2249 & 0.2297 & 0.2039 & 0.1842 & 0.188 & 0.1669 \\
\hline \multirow[t]{3}{*}{2} & $(5,7)$ & 0.4739 & 0.4085 & 0.3706 & 0.392 & 0.3426 & 0.3198 & 0.321 & 0.2805 & 0.2618 \\
\hline & $(7,9)$ & 0.4027 & 0.3625 & 0.3262 & 0.3264 & 0.2949 & 0.2753 & 0.2672 & 0.2414 & 0.2254 \\
\hline & $(10,12)$ & 0.3428 & 0.3289 & 0.2858 & 0.2613 & 0.2538 & 0.2369 & 0.214 & 0.2078 & 0.194 \\
\hline
\end{tabular}

Table 5: MSEs of Maximum Likelihood and Bayes estimates for $\alpha$ and $\lambda$ and the future $s$ th record value when $\lambda=1$

\begin{tabular}{|c|c|c|c|c|c|c|c|c|c|c|}
\hline \multirow[t]{2}{*}{$\alpha$} & \multirow[t]{2}{*}{$(m, s)$} & \multicolumn{3}{|c|}{ Maximum Likelihood } & \multicolumn{3}{|c|}{ Non-informative Bayes } & \multicolumn{3}{|c|}{ Informative Bayes } \\
\hline & & $\tilde{\alpha}$ & $\tilde{\lambda}$ & $r_{s}$ & $\hat{\hat{\alpha}}$ & $\hat{\hat{\lambda}}$ & $r_{s(B S)}$ & $\hat{\alpha}$ & $\hat{\hat{\lambda}}$ & $r_{s(B S)}$ \\
\hline \multirow[t]{3}{*}{0.5} & $(5,7)$ & 0.3459 & 0.2857 & 0.2595 & 0.2602 & 0.2274 & 0.2122 & 0.213 & 0.1862 & 0.1738 \\
\hline & $(7,9)$ & 0.2946 & 0.2579 & 0.2286 & 0.2166 & 0.1957 & 0.1827 & 0.1774 & 0.1602 & 0.1496 \\
\hline & $(10,12)$ & 0.2518 & 0.2267 & 0.2057 & 0.1734 & 0.1684 & 0.1572 & 0.142 & 0.1379 & 0.1287 \\
\hline \multirow[t]{3}{*}{1} & $(5,7)$ & 0.3712 & 0.3259 & 0.2819 & 0.2875 & 0.2642 & 0.2346 & 0.2354 & 0.2163 & 0.192 \\
\hline & $(7,9)$ & 0.3198 & 0.2895 & 0.2496 & 0.2394 & 0.2274 & 0.2019 & 0.196 & 0.1862 & 0.1653 \\
\hline & $(10,12)$ & 0.2625 & 0.2585 & 0.2258 & 0.1917 & 0.1957 & 0.1738 & 0.1569 & 0.1602 & 0.1423 \\
\hline \multirow[t]{3}{*}{2} & $(5,7)$ & 0.4218 & 0.3556 & 0.3257 & 0.3341 & 0.292 & 0.2725 & 0.2735 & 0.239 & 0.2231 \\
\hline & $(7,9)$ & 0.3635 & 0.3101 & 0.2865 & 0.2782 & 0.2513 & 0.2346 & 0.2277 & 0.2057 & 0.192 \\
\hline & $(10,12)$ & 0.2989 & 0.2748 & 0.2549 & 0.2227 & 0.2163 & 0.2019 & 0.1823 & 0.1771 & 0.1653 \\
\hline
\end{tabular}

Table 6: Efficiencies of Bayes estimates for $\alpha$ and $\lambda$ and the future $s$ th record value with respect to Maximum Likelihood estimates when $\lambda=0.5$

\begin{tabular}{|c|c|c|c|c|c|c|c|}
\hline \multirow[t]{2}{*}{$\alpha$} & \multirow[t]{2}{*}{$(m, s)$} & \multicolumn{3}{|c|}{ Non-informative Bayes } & \multicolumn{3}{|c|}{ Informative Bayes } \\
\hline & & eff $(\hat{\hat{\alpha}})$ & eff $(\hat{\hat{\lambda}})$ & eff $\left(r_{s(B S)}\right)$ & eff $(\hat{\hat{\alpha}})$ & eff $(\hat{\hat{\lambda}})$ & $e f f\left(r_{s(B S)}\right)$ \\
\hline \multirow[t]{3}{*}{0.5} & $(5,7)$ & 1.2542 & 1.2215 & 1.2055 & 1.5316 & 1.4915 & 1.4728 \\
\hline & $(7,9)$ & 1.2805 & 1.2647 & 1.2397 & 1.5642 & 1.5452 & 1.5145 \\
\hline & $(10,12)$ & 1.3740 & 1.3414 & 1.2813 & 1.6783 & 1.6391 & 1.5645 \\
\hline \multirow[t]{3}{*}{1} & $(5,7)$ & 1.2315 & 1.2003 & 1.1969 & 1.5038 & 1.4661 & 1.4618 \\
\hline & $(7,9)$ & 1.2569 & 1.2361 & 1.2216 & 1.5357 & 1.5094 & 1.4918 \\
\hline & $(10,12)$ & 1.3273 & 1.3287 & 1.2697 & 1.6205 & 1.6234 & 1.5512 \\
\hline \multirow[t]{3}{*}{2} & $(5,7)$ & 1.2089 & 1.1924 & 1.1588 & 1.4763 & 1.4563 & 1.4156 \\
\hline & $(7,9)$ & 1.2338 & 1.2292 & 1.1849 & 1.5071 & 1.5017 & 1.4472 \\
\hline & $(10,12)$ & 1.3119 & 1.2959 & 1.2064 & 1.6019 & 1.5828 & 1.4732 \\
\hline
\end{tabular}


Table 7: Efficiencies of Bayes estimates for $\alpha$ and $\lambda$ and the future $s$ th record value with respect to Maximum Likelihood estimates when $\lambda=0.5$

\begin{tabular}{|c|c|c|c|c|c|c|c|}
\hline \multirow[t]{2}{*}{$\alpha$} & \multirow[t]{2}{*}{$(m, s)$} & \multicolumn{3}{|c|}{ Non-informative Bayes } & \multicolumn{3}{|c|}{ Informative Bayes } \\
\hline & & $e f f(\hat{\hat{\alpha}})$ & eff $(\hat{\hat{\lambda}})$ & $e f f\left(r_{s(B S)}\right)$ & $e f f(\hat{\hat{\alpha}})$ & eff $(\hat{\hat{\lambda}})$ & eff $\left(r_{s(B S)}\right)$ \\
\hline \multirow[t]{3}{*}{0.5} & $(5,7)$ & 1.3294 & 1.2564 & 1.2229 & 1.6239 & 1.5344 & 1.4931 \\
\hline & $(7,9)$ & 1.3601 & 1.3178 & 1.2512 & 1.6607 & 1.6099 & 1.5281 \\
\hline & $(10,12)$ & 1.4521 & 1.3462 & 1.3085 & 1.7732 & 1.6439 & 1.5983 \\
\hline \multirow[t]{3}{*}{1} & $(5,7)$ & 1.2911 & 1.2335 & 1.2016 & 1.5769 & 1.5067 & 1.4682 \\
\hline & $(7,9)$ & 1.3358 & 1.2731 & 1.2363 & 1.6316 & 1.5548 & 1.5100 \\
\hline & $(10,12)$ & 1.3693 & 1.3209 & 1.2992 & 1.6730 & 1.6136 & 1.5868 \\
\hline \multirow[t]{3}{*}{2} & $(5,7)$ & 1.2625 & 1.2178 & 1.1952 & 1.5422 & 1.4879 & 1.4599 \\
\hline & $(7,9)$ & 1.3066 & 1.2340 & 1.2212 & 1.5964 & 1.5075 & 1.4922 \\
\hline & $(10,12)$ & 1.3422 & 1.2705 & 1.2625 & 1.6396 & 1.5517 & 1.5420 \\
\hline
\end{tabular}

\section{References}

[1] K.N. Chandler, "The distribution and frequency of record values", J. R. Stat. Soc. Ser. B 14(2), 220-228, 1952.

[2] M. Ahsanullah, "Linear prediction of record values for the two parameter exponential distribution". Annals of the Institute of Statistical Mathematics, 32, 363-368, 1980

[3] M. Ahsanullah, "Introduction to Record Values". Ginn Press, Needham Heights, Massachusetts, 1988.

[4] M. Ahsanullah, "Estimation of the parameters of the Gumbel distribution based on the m record values". Computational Statistics Quarterly, 3, 231-239, 1990 .

[5] M. Ahsanullah, "Record values. The Exponential Distribution: Theory, Methods and Applications", eds. N. Balakrishnan and A. P. Basu, Gordon and Breach Publishers, Newark, New Jersey. 1995.

[6] B. C. Arnold, N. Balakrishnan, and H. N. Nagaraja, "A First Course in Order Statistics", John Wiley \& Sons, New York, 1992.

[7] B. C. Arnold, N. Balakrishnan, and H. N. Nagaraja), "Records", John Wiley \& Sons, New York, 1998.

[8] N. Balakrishnan, M. Ahsanullah, and P. S. Chan, "Relations for single and product moments of record values from Gumbel distribution", Statistics and Probability Letters, 15, 223-227, 1992.

[9] N. Balakrishnan, and M. Ahsanullah, "Recurrence relations for single and product moments of record values from generalized Pareto distribution", Communications in Statistics-Theory and Methods, 23, 2841-2852, 1994.

[10] N. Balakrishnan, and M. Ahsanullah, "Relations for single and product moments of record values from exponential distribution", Journal of Applied Statistical Science, 2, 73-87, 1995.

[11] N. Balakrishnan, M. Ahsanullah, and P. S. Chan, "On the logistic record values and associated inference", Journal of Applied Statistical Science, 2, 233-248. 1995

[12] M. A. Selim, "Bayesian Estimations from the Two-Parameter Bathtub- Shaped Lifetime Distribution Based on Record Values", 8(2), 155-165, 2012.

[13] M. Nadar, A. Papadopoulos and F. Kizılaslan, "Statistical analysis for Kumaraswamy’s distribution based on record data", Stat Papers, 54(2), 335-369, 2013.

[14] M. Amini and N. Balakrishnan, "Nonparametric meta-analysis of independent samples of records", Computational Statistics \& Data Analysis, 66, 70-81, 2013

[15] M. Juhas and V. Skrivankova, "Characterization of general classes of distributions based on independent property of transformed record values", Applied Mathematics and Computation, 226, 44-50, 2014.

[16] N. Balakrishnan, P. S. Chan, and M. Ahsanullah, "Recurrence relations for moments of record values from generalized extreme value distribution", Communications in Statistics-Theory and Methods, 22, 1471-1482, 1993.

[17] M. Q. Shahbaz, S. Shahbaz and N. S. Butt, "The Kumaraswamy-Inverse Weibull Distribution", Pakistan journal of statistics and operation research, 8(3): 479-489, 2012.

[18] P. Basak and N. Balakrishnan, "Maximum likelihood prediction of future record statistic", Mathematical and statistical methods in reliability. In: Lindquist BH, Doksun KA (eds) Series on quality, reliability and engineering statistics, World Scientific Publishing, Singapore, 7, 159$175,2003$.

[19] E. K. Al-Hussaini and Z. F. Jaheen, "Bayesian prediction bounds for the Burr Type XII failure model", Commun Stat Theor Meth, 24, 18291842, 1995.

[20] P. Congdon, "Bayesian Statistical Modeling", Wiley, New York, 2001 